\title{
TERRITORIALISATION OU SPATIALISATION : LES AGENCES ET COMITÉS LOCAUX DE L'EAU AU BURKINA FASO
}

\author{
Jean-Philippe Venot et al.
}

Belin | L'Espace géographique

\section{4/2 - Tome 43 \\ pages 148 à 163}

\section{ISSN 0046-2497}

Article disponible en ligne à l'adresse:

http://www.cairn.info/revue-espace-geographique-2014-2-page-148.htm

Pour citer cet article :

Venot Jean-Philippe et al., « Territorialisation ou spatialisation : les agences et comités locaux de l'eau au Burkina Faso »,

L'Espace géographique, 2014/2 Tome 43, p. 148-163.

Distribution électronique Cairn.info pour Belin.

(C) Belin. Tous droits réservés pour tous pays.

La reproduction ou représentation de cet article, notamment par photocopie, n'est autorisée que dans les limites des conditions générales d'utilisation du site ou, le cas échéant, des conditions générales de la licence souscrite par votre établissement. Toute autre reproduction ou représentation, en tout ou partie, sous quelque forme et de quelque manière que ce soit, est interdite sauf accord préalable et écrit de l'éditeur, en dehors des cas prévus par la législation en vigueur en France. II est précisé que son stockage dans une base de données est également interdit. 


\section{Gestion locale de l'eau}

p. 148-163

\section{Territorialisation ou spatialisation : les agences et comités locaux de l'eau au Burkina Faso}

\author{
Jean-Philippe VenOT \\ IRD-UMR Gouvernance, risque, \\ environnement, développement (GRED) \\ 911 avenue d'Agropolis, BP 64501 \\ 34394 Montpellier CEDEX 5 \\ France \\ jean-philippe.venot@ird.fr
}

\author{
Bio Mohamadou \\ TOROU \\ Université de Ouagadougou \\ International Water \\ Management Institute (IWMI) \\ BP 7021, Ouagadougou, Burkina Faso
}

William's D A RE
Direction régionale Afrique
de l'Ouest continentale
UPR Green, CIRAD
Avenue Kennedy, 01BP596
Ouagadougou, Burkina Faso
williams.dare@cirad.fr
RÉSUMÉ - - En lien avec le discours dominant le secteur de l'eau, le Burkina Faso s'est engagé depuis vingt ans dans une politique de gestion intégrée des ressources en eau. Le fort dynamisme institutionnel observé marque une spatialisation de la gestion des ressources en eau, sur la base des limites hydrographiques des bassins versants. L'approche adoptée reste cependant descendante et ne permet pas l'expression des perceptions et actions locales. Accompagner les dynamiques ascendantes apparaît comme une condition nécessaire de la territorialisation, donc de la durabilité, des politiques et des modes de gestion des ressources en eau.

AFRIQUE DE L'OUEST, EAU, ESPACE GÉOGRAPHIQUE, GESTION DES RESSOURCES, TERRITOIRE
ABSTRACT .- Constructing space: Water agencies and committees in Burkina Faso Over the last 20 years, Burkina Faso has engaged in reforms promoting integrated water resources management that are in line with dominant water discourses. The strong institutional drive outlines a spatialisation of water management, based on the watershed's hydrographic boundaries. The approach however remains top-down and does not acknowledge local views and efforts. There is a need to support local initiatives as they play a crucial role in constructing space, hence sustainably shaping the ways water resources are used and governed.

PLACE, RESOURCES MANAGEMENT, SPACE, WATER, WEST AFRICA

\section{Introduction}

Depuis l'émergence de la notion de développement durable au sommet de la terre à Rio de Janeiro en 1992, la gestion intégrée des ressources en eau (GIRE) s'est progressivement imposée comme étant la réponse principale aux problèmes du secteur de l'eau. La gestion intégrée des ressources en eau reposerait sur quatre principes directeurs: l'existence d'une échelle optimale de gestion, le bassin versant; la nécessité d'une approche démocratique de gestion des ressources en eau (prenant souvent une forme particulière de participation, les 
plates-formes multi-acteurs); la reconnaissance du rôle central des femmes dans la gestion des ressources en eau; et le besoin de reconnaître l'eau comme un bien économique et de la gérer en conséquence (Gwp, 2000).

La littérature sur la gestion intégrée des ressources en eau, qu'elle soit scientifique ou pratique, est vaste. Le débat s'est notamment centré sur la polysémie du terme même, la gestion intégrée des ressources en eau pouvant s'entendre comme philosophie, principe, cadre méthodologique, approche, processus ou prescription (Molle, 2012); sur l'intérêt théorique de l'idée d'intégration, rarement remise en cause; et sur les difficultés de sa mise en pratique (Chéné, 2009), notamment dans les pays en développement (Shah, van Koppen, 2006). Les difficultés d'application de la gestion intégrée des ressources en eau seraient notamment liées à l'inadaptation $\mathrm{du}$ modèle aux réalités des secteurs de l'eau des pays en développement, à la standardisation et à la rigidité d'application de "bonnes pratiques " qui ne répondraient pas aux besoins immédiats des populations (que serait l'augmentation de l'offre en eau), et à un manque de volonté politique réelle d'accompagner des réformes qui seraient lancées sous pression extérieure (Lankford, Hepworth, 2010; Bouquet, 2012). De nombreux auteurs soulignent aussi que le bassin versant, bien que présenté comme entité " naturelle " de gestion, constitue une structuration politique de l'espace qui résulte des choix de certains acteurs (Blomquist, Schlager, 2005; Ghiotti, 2006; Warner et al., 2008; Graefe, 2011).

La dichotomie entre théorie et mise en pratique de la gestion intégrée des ressources en eau que de nombreux auteurs constatent, ne pourra pas, nous semble-til, être résolue alors même que «la pratique de la gestion intégrée des ressources en eau est et sera probablement perpétuellement contestée "(Molle, 2012, p. 36). Nous ne traitons donc pas de la gestion intégrée des ressources en eau à un niveau conceptuel $^{1}$, mais nous nous intéressons à des données empiriques sur les politiques de l'eau et leur mise en pratique dans un pays d'Afrique de l'Ouest, le Burkina Faso, l'un des premiers pays du continent à réformer son secteur de l'eau suivant les principes de la gestion intégrée des ressources en eau, et ce dès le milieu des années 1990 (Petit, Baron, 2009; Cherlet, Venot, 2013). Nous contribuons ainsi à une lecture de la gestion intégrée des ressources en eau qui, au-delà des déclarations globales, se veut pragmatique et centrée sur les pratiques d'acteurs.

Plus spécifiquement, cet article vise à répondre à la question suivante: dans quelle mesure les approches adoptées au Burkina Faso pour la mise en place de nouvelles structures de gestion de l'eau ont-elles permis le démarrage de dynamiques endogènes d'appropriation par les acteurs locaux? Nous partons, en effet, de l'hypothèse que la démarche de création de ces nouveaux organes de gestion joue un rôle crucial dans leur appropriation (ou non) et donc leur niveau de fonctionnement à moyen et long terme. Nous analyserons ainsi le processus de création des agences de l'eau (section 2) et des comités locaux de l'eau (section 3). En nous inscrivant dans le champ de la géographie sociale qui souligne que tout territoire se caractérise par une dimension politique d'institutionnalisation de l'espace et d'une dimension ascendante s'inscrivant à la fois dans l'affectif et l'action (D'Aquino, 2002; Di Méo, Buléon, 2005), nos résultats empiriques permettent de nourrir une réflexion plus large sur la territorialisation (ou non) des politiques de l'eau au Burkina Faso (section 4).
1. François Molle (2012) présente une analyse critique du concept et de ses incohérences. 
2. Voir Petit, Baron (2009) et Cherlet, Venot (2013) pour une description détaillée des justifications et des individus impliqués dans ces processus.

3. En septembre 2013 seules les agences de I'eau du Nakambé et du Mouhoun et Bannifing avaient été créées (en 2007 et 2013, respectivement). Le processus de création des agences de la Comoé, du Liptako et du Gourma était lancé par la mise en place de directions générales au sein du ministère en charge des ressources en eau.

\section{Une institutionnalisation précoce du bassin versant comme unité de gestion}

La première politique nationale de l'eau burkinabé fut élaborée après la vague de sécheresses qui frappa le Sahel dans le milieu des années 1970. Cette politique mettait l'accent sur la construction d'infrastructures hydrauliques. Les révisions successives effectuées dans les années 1980 ne modifièrent que très peu l'essence de cette politique jusqu'à ce que l'État, à la suite des plans d'ajustements structurels des années 1990, ne se désengage de la plupart des secteurs dont celui de l'eau. Par la suite, et dès le milieu des années 1990, le Burkina Faso, avec le soutien de la coopération danoise, produisit une série de réformes inspiréee des principes de la gestion intégrée des ressources en eau (GIRE). Ces réformes aboutirent à l'adoption de la "Loi d'orientation relative à la gestion de l'eau » en 2001 (Gouvernement du Burkina Faso, 2001) et à sa traduction dans un "Plan d'action pour la gestion intégrée des ressources en eau " (PAGIRE) en 2003 (Gouvernement du Burkina Faso, 2003) qui constitue le cadre national de la planification, la gestion et l'utilisation des ressources en eau à l'horizon $2015^{2}$. En lien avec les principes de la gestion intégrée des ressources en eau, les politiques et stratégies en matière d'eau considèrent le bassin hydrographique comme "le cadre approprié de planification et de gestion de la ressource [en eau]" (Gouvernement du Burkina Faso, 2001) et visent à ériger celui-ci en nouveau territoire en créant de nouvelles structures de gestion sur le modèle français: les agences de l'eau (à l'échelle des bassins versants nationaux) et les comités locaux de l'eau (CLE) à l'échelle des sous-bassins.

Le discours et les textes réglementaires mettent en avant le bassin versant comme seul et unique territoire de l'eau, mais la réalité apparaît plus complexe montrant que les principes s'adaptent à la réalité du terrain et aux contraintes administratives et biophysiques. Outre le bassin versant, une autre division de l'espace a, en effet, été introduite au niveau national: l'espace de gestion à l'échelle duquel les agences de l'eau sont actuellement mises en place ${ }^{3}$.

La figure 1 laisse voir que ces espaces de gestion ne recoupent que partiellement les limites hydrographiques. Par exemple, l'espace de gestion du Mouhoun et Bannifing inclut une portion de l'extrême amont du bassin versant du Niger. L'espace de gestion du Nakambé ne représente qu'une portion du bassin versant homonyme, les affluents situés à l'est du bras principal (et ne le rejoignant que plus en aval, au Ghana) ayant été inclus dans l'espace de gestion du Gourma (lui-même portion du bassin versant du Niger). Enfin, l'espace de gestion du Liptako, situé au nord-est du pays, recouvre les affluents nord du Niger, qui ne rejoignent les affluents situés plus au sud (Gourma) qu'en dehors du Burkina Faso (au Niger).

Les espaces de gestion ont été délimités sur la base des points de confluence et constituent des entités cohérentes pour les décideurs burkinabé - notamment dans l'optique d'une opérationnalisation de la gestion intégrée des ressources en eau. Ces adaptations reflètent une appropriation du concept de bassin versant, appropriation qui reste cependant circonscrite aux services administratifs du ministère de l'Agriculture, de l'Hydraulique et des Ressources halieutiques (MAHRH).

Le processus de création des agences de l'eau traduit cet état de fait. Sur le modèle français, les agences de l'eau sont, par statut, constituées de trois organes: une direction générale du ministère de l'Agriculture, de l'Hydraulique et des Ressources halieutiques (depuis 2013, le ministère de l'Eau, des Aménagements hydraulique et de 


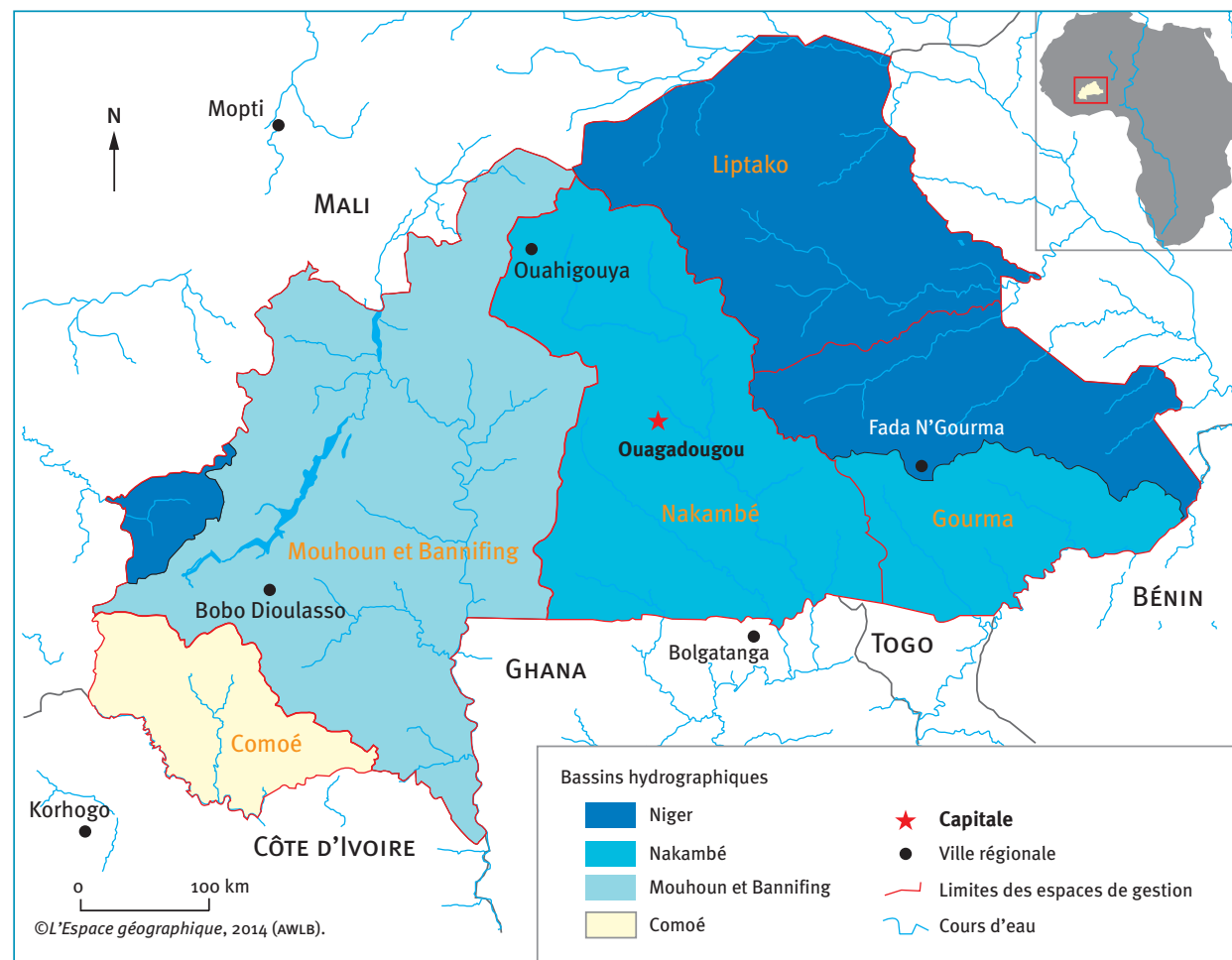

Fig. 1/ Bassin versant et espaces de gestion au Burkina Faso
l'Assainissement) ${ }^{4}$, un comité de bassin, et un conseil d'administration. Le comité de bassin, instance délibérative de l'agence, est constitué de représentants de trois collèges, en nombre égal: l'administration (fonctionnaires de différents ministères et représentants du gouvernement aux échelles régionales, provinciales et/ou départementales), les collectivités territoriales (régionales et/ou communales) et un collège vaguement défini comme étant celui des "usagers ». La participation effective de ces différents acteurs aux délibérations et leur représentativité dépendent du processus ayant abouti à leur sélection.

Ce processus soulève cependant de nombreuses questions comme l'illustre la sélection des membres du comité de bassin de l'agence de l'eau du Mouhoun et Bannifing. Le processus de sélection était coordonné par un "noyau technique " du ministère de l'Agriculture, de l'Hydraulique et des Ressources halieutiques avec l'appui d'un bureau d'étude danois (COWI), très actif dans le secteur et sur l'ensemble de l'Afrique de l'Ouest. Selon les mots du coordonnateur du noyau technique (devenu depuis directeur général de l'agence), "la sélection des représentants de l'administration et des collectivités territoriales s'est bien passée; en ce qui concerne les usagers, c'est un peu difficile [sic] " (encadré 1).

Il apparaît que l'institutionnalisation de la gestion intégrée des ressources en eau et du bassin versant comme unité de gestion se soit faite de façon descendante, avec un fort investissement des services du ministère de l'Agriculture, de l'Hydraulique et des Ressources halieutiques ce qui explique l'adaptation des frontières des agences de l'eau aux contraintes administratives. Les représentants des "usagers " au sein des agences de l'eau apparaissent représentatifs et légitimes vis-à-vis de l'administration
4. À la suite des élections législatives et municipales de décembre 2012,

un nouveau ministère de l'Eau,

des Aménagements hydrauliques et de l'Assainissement a été créé alors que l'eau agricole relevait du ministère de l'Agriculture, de I'Hydraulique et des Ressources halieutiques depuis 2002 et l'eau potable et

I'assainissement relevaient du portefeuille de multiples ministères et agences. 
Encadré 1 / Description du processus d’identification des représentants des usagers au sein du comité de bassin de l'agence de l'eau du Mouhoun et Bannifing

En février 2012, le «noyau technique» de l'agence de l'eau du Mouhoun et Bannifing a invité par l'intermédiaire des directions régionales du ministère de l'Agriculture, de l'Hydraulique et des Ressources halieutiques une cinquantaine de personnes à partir d'un diagnostic initial qui identifiait les principaux utilisateurs en eau du bassin versant. L'objectif de ce " forum des usagers » était de choisir leurs représentants au sein du comité de bassin sur les termes de référence, qui, comme le diagnostic, avaient été développés par le noyau technique du ministère de l'Agriculture, de l'Hydraulique et des Ressources halieutiques avec l'appui de COWI. Les termes de référence proposaient des critères de sélection et une première liste de 15 délégués potentiels. La durée de la réunion (une demi-journée) n’a malheureusement pas permis de débattre du bien-fondé de cette liste. Les irrigants de la vallée du Sourou (une zone de production rizicole intensive), bien que s'estimant sous-représentés, ne sont pas parvenus, par exemple, à obtenir de siège supplémentaire et ce malgré les fortes tensions apparues. En outre, aucun participant au forum n'était originaire de la région du Sud-Ouest (dont plus de $90 \%$ se trouve dans le bassin versant du Mouhoun ce qui correspond à $15 \%$ de la superficie de ce dernier) mais cela n'a pas empêché de compter, dans la liste définitive, trois représentants issus de cette région - sur la base du diagnostic initial. Cette liste comprend sept utilisateurs industriels (industries minières, cotonnières et sociétés de production d'eau potable et d'assainissement), un concessionnaire de chasse, une organisation non gouvernementale, une agence parapublique agricole, et cinq représentants d'organisations paysannes. Enfin, et même si seulement deux comités locaux de l'eau (CLE) existaient à la date de cette réunion, l'absence de représentants de ces comités locaux de l'eau (pourtant organes statutaires de l'agence de l'eau) est à noter et démontre que l'articulation entre agences et comités locaux de l'eau reste balbutiante.

(et des bureaux d'études qui ont accompagné le processus de mise en place) mais la majorité des utilisateurs d'eau, à savoir les populations rurales, et les comités locaux de l'eau n'ont que très peu contribué à leur identification.

\section{À l'épreuve du local : la mise en pratique d'une politique}

\section{Logique et méthodologie}

Au cours des dix dernières années, le gouvernement et ses principaux partenaires du secteur de l'eau ont commandité de nombreuses études sur le niveau d'avancement de la gestion intégrée des ressources en eau au Burkina Faso. Ces études s'accordent pour souligner le retard enregistré dans la mise en place de nouveaux organes de gestion (agences de l'eau et comités locaux de l'eau) et surtout leur inertie et leur manque de résultats en termes de meilleure gestion de l'eau et de lutte contre la pauvreté, et ce, près de dix ans après les premières tentatives de structuration de la gestion de l'eau sur la base de limites hydrographiques.

Ces études se limitent cependant à constater l'absence d'actions pratiques menées sur le terrain mais sans en déterminer les causes profondes, au-delà des coupables habituels - manque de moyens humains et financiers -, généralement dénoncés par les autorités administratives et les usagers (DGRE, 2006; DGRE et al., 2008; GWP/AO, 2009; MAHRH, 2010). En ce qui concerne les comités locaux de l'eau, il est certes dit ou écrit que les "démarches employées [pour leur création] n'ont pas eu les résultats escomptés"(BEGE, DGRE, 2008, p. 70) mais la stratégie adoptée reste peu critiquée et, toujours selon ces études, " apparaît pertinente " (BEGE, DGRE, 2008, 
p. 48). Nous partageons le diagnostic d'incapacité des comités locaux de l'eau qui a suivi leur reconnaissance officielle par les autorités administratives du ministère de l'Agriculture, de l'Hydraulique et des Ressources halieutiques et le gouvernement burkinabè il y a 5 à 10 ans. Nous faisons l'hypothèse que cette incapacité est étroitement liée au processus de mise en place de ces structures de gestion, processus que nous analyserons en détail.

L'étude a été menée en deux phases successives entre mars 2011 et septembre 2012. Dans un premier temps (mars-juin 2011), une soixantaine d'entretiens nous a permis de mieux cerner les dynamiques de mise en place de dix comités locaux de l'eau dans le Nakambé. Outre les responsables de ces comités locaux de l'eau et des bureaux d'études ayant coordonné leur mise en place, nous avons rencontré les services déconcentrés du ministère de l'Agriculture, de l'Hydraulique et des Ressources halieutiques, des responsables administratifs (préfets et haut-commissaires), des responsables d'organisations non gouvernementales, d'associations et d'organisations paysannes, ainsi que des élus locaux (à l'échelle du village et de la commune) dans les zones couvertes par les comités locaux de l'eau. Dans un second temps (février-septembre 2012), des informations complémentaires ont été obtenues pour trois comités locaux de l'eau, dont deux situés dans le bassin du Mouhoun (Bougouriba 7 et Kou) et un dans celui du Nakambé (Itenga). Pour chacun de ces comités locaux de l'eau, une cinquantaine d'entretiens ont été conduits auprès des acteurs susmentionnés tout en privilégiant les membres des instances de ces trois comités locaux de l'eau. Cette analyse des dynamiques locales est enrichie par une étude bibliographique ainsi que par divers entretiens et échanges réguliers avec des décideurs nationaux du ministère de l'Agriculture, de l'Hydraulique et des Ressources halieutiques, des responsables des agences de l'eau et d'autres acteurs du secteur (bailleurs de fonds, ONG, chercheurs).

\section{Des ajustements mais une approche largement incontestée}

En 2012, le Burkina Faso comptait 35 comités locaux de l'eau (CLE) ${ }^{5}$. Dans les bassins du Niger et du Nakambé, le processus de mise en place a été coordonné soit par la Direction générale de l'inventaire des ressources hydrauliques (DGIRH) ${ }^{6} \mathrm{du}$ ministère de l'Agriculture, de l'Hydraulique et des Ressources halieutiques directement (3 comités locaux de l'eau dans le bassin du Nakambé et 3 comités locaux de l'eau dans le bassin du Niger, en 2004-2005), soit par des maîtrises d'œuvres sociales (MOS) diligentées par la Direction générale de l'inventaire des ressources hydrauliques (20 comités locaux de l'eau en 2005-2006), soit par des opérateurs privés (5 comités locaux de l'eau). Entre 2008 et 2011, les services déconcentrés du ministère de l'Agriculture, de l'Hydraulique et des Ressources halieutiques ont coordonné la création de deux comités locaux de l'eau dans le bassin du Mouhoun et, avec l'appui d'une association, la restructuration de deux autres dans les bassins du Mouhoun et de la Comoé.

Alors que les comités locaux de l'eau sont, dans l'esprit de la législation, des cadres de concertation au niveau des bassins versants locaux (BEGE, DGRE, 2008), seuls les quatre derniers comités locaux de l'eau mis en place et localisés dans le bassin du Mouhoun et de la Comoé ont été pensés sur la base de limites hydrographiques. Leur création s'est appuyée sur l'identification de leur "zone de compétence " par les services de l'agence de l'eau ou du ministère de l'Agriculture, de l'Hydraulique et des Ressources halieutiques. Les comités locaux de l'eau mis en place quelques années auparavant dans les bassins du Niger et du Nakambé ont, au contraire, été pensés pour
5. Depuis 2012, le bureau d'étude danois COWI appuie le gouvernement du Burkina Faso pour la création d'une vingtaine de nouveaux comités locaux de l'eau dans le bassin versant du Mouhoun.

6. Devenue Direction générale des Ressources en eau (DGRE) en 2006. 
la gestion de points d'eau naturels ou artificiels (barrages). Les sites d'implantation ayant été choisis par la Direction générale de l'inventaire des ressources hydrauliques sur deux critères principaux, à savoir la présence de ressources en eau à valoriser et protéger et l'existence (ou le risque) de conflits d'usages. Ces comités locaux de l'eau impliquent (du moins en théorie) des acteurs des villages, communes, voire des provinces situées autour de ces points d'eau sans pour autant que la participation des acteurs n'ait été circonscrite à un espace dont la frontière eût été formellement identifiée. Ce centrage des comités locaux de l'eau autour de points d'eau, perçus comme de «puissants facteurs de mobilisation, d'adhésion et de participation effective des populations " (BEGE, DGRE, 2008, p. 54) et qui constituent des lieux où se cristallisent les interactions entre différents acteurs, porte les germes d'une possible appropriation de ces nouvelles structures de gestion par les acteurs locaux. À l'inverse, "les notions de bassin versant et de sous bassin versant restent encore méconnues " (ibidem, p. 52) et il reste difficile de mobiliser les acteurs pour une gestion à une échelle plus vaste que celle de l'espace «autour» d'un point d'eau (entretien avec le directeur général de l'agence de l'eau du Nakambé). Les contraintes financières et de coordination (il existe plus de 1200 points d'eau au Burkina Faso: Venot, Hirvonen, 2013) rendent cependant nécessaire, selon l'administration, d'étendre la zone de compétence des comités locaux de l'eau du point d'eau au bassin versant local (d'une superficie d'environ $3000 \mathrm{~km}^{2}$ ).

C'est l'une des modifications introduites par la nouvelle version du «Document Guide de conception, création et fonctionnement " des comités locaux de l'eau formulée en 2010 (DGRE, 2010) après une étude commanditée par le ministère de l'Agriculture, de l'Hydraulique et des Ressources halieutiques et visant à "tirer les leçons de la mise en place des Cle "(Bege, Dgre, 2008). Le nouveau guide insiste sur le besoin d'accompagnement et précise les étapes à suivre pour la mise en place des comités locaux de l'eau mais ne diffère que très peu, sur le fond, de l'ancienne version (DGIRH, 2004). Aucune réflexion sur la pertinence du bassin versant local comme "unité de base " de gestion des ressources en eau n'est menée, et les acteurs locaux restent vus comme des bénéficiaires de l'approche qu'il faudrait sensibiliser et mobiliser plutôt que des partenaires à part entière à impliquer dans la réflexion.

\section{Des maîtrises d'œuvres sociales sélectionnées mais peu impliquées}

Dans le Nakambé, le processus de mise en place des comités locaux de l'eau a été coordonné par des maîtrises d'œuvres sociales (MOS) recrutées par la Direction générale de l'inventaire des ressources hydrauliques dans le courant de l'année 2005. Il s'agit d'ONG, d'associations de droit burkinabé, de bureaux d'études ou de cabinets de conseil qui souvent ont été créés par (ou comptent parmi leurs membres) des anciens fonctionnaires du ministère de l'Agriculture, de l'Hydraulique et des Ressources halieutiques qui ont gardé des liens étroits avec l'administration.

Les organisations ayant manifesté leur intérêt ont d'abord été présélectionnées et ont bénéficié d'une formation à la gestion intégrée des ressources en eau et la stratégie envisagée pour la création des comités locaux de l'eau. Par la suite, elles ont été conviées à soumettre des offres techniques et financières, selon des termes de référence définis par la Direction générale de l'inventaire des ressources hydrauliques. Outre la qualité des offres, la connaissance de la zone d'intervention (et des acteurs à impliquer) et la familiarité avec les notions de gestion intégrée des ressources en eau étaient censées être des critères primordiaux de sélection (BEGE, DGRE, 2008). 
Dans la pratique, il apparaît que la plupart des maîtrises d'œuvres sociales sélectionnées avaient une expérience dans l'animation et la sensibilisation en milieu paysan ou dans le renforcement des capacités mais sans réel lien avec la gestion des ressources en eau. Dans ces conditions, la formation initiale revêtait une importance particulière. Cependant, la plupart des intervenants sur le terrain n'ont pu bénéficier de cette formation qui s'adressait principalement aux responsables des maitrises d'œuvres sociales. En outre, la formation d'une durée de deux jours a par la suite été jugée trop courte et trop générale par l'ensemble des acteurs, les personnes en ayant bénéficié considérant qu'elles n'avaient pas été outillées pour appréhender la complexité des dynamiques locales et mener à bien leurs tâches.

Enfin, le niveau de compétence, d'implication et l'intérêt que les maîtrises d'œuvres sociales avaient pour le sujet et l'approche étaient très variables (voir aussi Bege, DGRe, 2008) 7 . Nos enquêtes révèlent que les motivations premières de ces organisations étaient l'acquisition de nouvelles connaissances par le moyen de la formation, et l'extension de leur domaine de compétence. Jamais la participation des acteurs locaux à la gouvernance des ressources en eau ne nous a été présentée comme une raison de leur implication dans le processus.

\section{Étapes et contraintes de la mise en place des comités locaux de l'eau (CLE)}

La construction territoriale s'inscrit dans un temps long (Di Méo, Buléon, 2007). Pourtant, le temps imparti à la mise en place des comités locaux de l'eau (trois mois) et le temps prévu pour leur accompagnement initial (trois mois supplémentaires et rarement réalisé) ont constitué une entrave majeure à l'établissement de conditions favorables à l'émergence de dynamiques endogènes sans lesquelles les comités locaux de l'eau ne sauraient s'inscrire dans une certaine durabilité d'action. Le processus suivi suscite également de nombreuses questions en ce qui concerne la tenue des réunions et la sélection des participants aux comités locaux de l'eau. Le tableau 1 illustre l'écart entre le processus, tel que planifié par les deux guides successifs, et les pratiques observées et décrites en détail.

La phase de diagnostic (étape 2, tableau 1) et les premières sensibilisations (étape 3, figure 2) étaient censées favoriser l'émergence d'une vision partagée de l'eau et des problématiques y afférant dans la zone considérée afin d'ouvrir la voie pour la création d'une structure qui répondrait à ces défis locaux: le comité local de l'eau. Cependant, nos enquêtes révèlent que les populations n'ont été que marginalement impliquées dans cette phase initiale; le dialogue s'établissant entre les maîtrises d'œuvres sociales, les services techniques et les autorités administratives. Les premières réunions de sensibilisation (étape 3, tableau 1) qui auraient pu permettre aux acteurs locaux d'exprimer leurs préoccupations et attentes se sont limitées à un apport d'informations sur la notion de gestion intégrée des ressources en eau et sur un état des lieux réalisé par des acteurs extérieurs. Les diagnostics ont été limités à une description du milieu physique et social (sur la base de quelques enquêtes de responsables locaux) et à un inventaire des ressources en eau et des problématiques de gestion. Ils proposent des solutions génériques (mise en place d'une zone de marnage, plantations antiérosive, réhabilitation des infrastructures hydrauliques, etc.).

Les réunions de concertation suivantes (étape 4, tableau 1) visaient à identifier l'ensemble des acteurs pouvant contribuer aux travaux du comité local de l'eau et à mettre en place un comité multipartite pour l'élaboration de ses textes statutaires
7. Parmi les douze maîtrises d'œuvres sociales sélectionnées pour la mise en place des comités locaux de l'eau dans le Nakambé, quatre avaient disparu et cinq changé de personnel lorsque nous avons réalisé nos enquêtes. Dans ces neuf cas, la documentation afférente au processus n'était plus disponible. Ceci illustre clairement la volatilité des structures et leur manque d'expérience. 


\section{Tabl. 1/ Activités envisagées et effectuées pour la mise en place des Comités locaux de l’eau}

\begin{tabular}{|c|c|c|c|c|c|}
\hline & Étape 1: préparation & Étape 2 : diagnostic & $\begin{array}{c}\text { Étape 3: } \\
\text { sensibilisation }\end{array}$ & $\begin{array}{l}\text { Étape } 4 \text { : concertation } \\
\text { et désignation }\end{array}$ & $\begin{array}{c}\text { Étape } 5: \\
\text { officialisation }\end{array}$ \\
\hline Guide 2004 & $\begin{array}{l}\text { Dossier préparatoire } \\
\text { soumis à } \\
\text { l'administration. }\end{array}$ & $\begin{array}{l}\text { Consultation } \\
\text { des villages-communes } \\
\text { par l'administration*. }\end{array}$ & 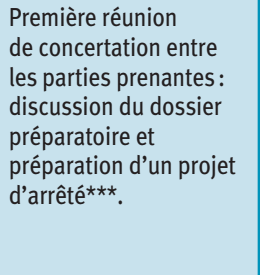 & $\begin{array}{l}\text { Seconde réunion } \\
\text { de concertation: } \\
\text { discussion, ajustement } \\
\text { et adoption du projet } \\
\text { d'arrêté; désignation } \\
\text { des représentants au } \\
\text { sein du comité local } \\
\text { de l'eau par les trois } \\
\text { collèges }^{\star \star \star \star \star} \text {. }\end{array}$ & $\begin{array}{l}\text { Signature de l'arrêté } \\
\text { par l'administration } \\
\text { et session officielle } \\
\text { d'installation sous } \\
\text { son patronage. }\end{array}$ \\
\hline Guide 2010 & $\begin{array}{l}\text { Dossier préparatoire } \\
\text { soumis à l'agence de } \\
\text { l'eau et amendement en } \\
\text { accord avec les services } \\
\text { déconcentrés du } \\
\text { ministère de } \\
\text { l'Agriculture, } \\
\text { de l'Hydraulique et des } \\
\text { Ressources halieutiques. } \\
\text { Proposition et mise } \\
\text { en place d'un comité } \\
\text { technique de suivi par } \\
\text { l'administration. }\end{array}$ & $\begin{array}{l}\text { Diagnostic conjoint } \\
\text { (services techniques, } \\
\text { communautés, } \\
\text { promoteur) et } \\
\text { proposition pour } \\
\text { le quota d'acteurs } \\
\text { par collège } e^{\star \star} \text {. }\end{array}$ & $\begin{array}{l}\text { Trois réunions de } \\
\text { sensibilisation sur } \\
\text { la gestion intégrée } \\
\text { des ressources en eau, } \\
\text { les enjeux locaux de } \\
\text { l'eau et le comité local } \\
\text { de l'eau. } \\
\text { Élaboration des textes } \\
\text { constitutifs du comité } \\
\text { local de l'eau par une } \\
\text { commission de } \\
\text { rédaction, appuyée } \\
\text { par le comité technique } \\
\text { de suivi }\end{array}$ & 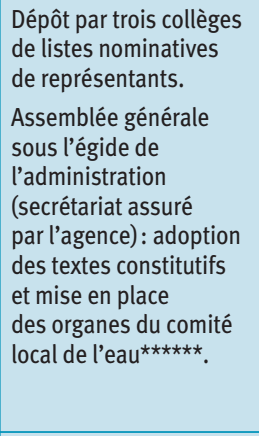 & $\begin{array}{l}\text { Signature de l'arrêté } \\
\text { par l'administration } \\
\text { et session officielle } \\
\text { d'installation sous } \\
\text { son patronage. } \\
\text { Accompagnement du } \\
\text { comité local de l'eau. }\end{array}$ \\
\hline $\begin{array}{l}\text { Activités effectuées } \\
\text { par les maîtrises } \\
\text { d'œuvres sociales }\end{array}$ & & $\begin{array}{l}\text { Documentation et } \\
\text { entretiens avec } \\
\text { les autorités locales } \\
\text { (chefs coutumiers et } \\
\text { collectivités territoriales) } \\
\text { et les services } \\
\text { techniques } \\
\text { déconcentrés } \\
\text { du ministère de } \\
\text { l'Agriculture, } \\
\text { de l'Hydraulique et des } \\
\text { Ressources halieutiques. }\end{array}$ & $\begin{array}{l}\text { Première réunion: } \\
\text { restitution du diagnostic } \\
\text { aux parties prenantes. }\end{array}$ & $\begin{array}{l}\text { Rédaction ad-hoc } \\
\text { des textes statutaires } \\
\text { (par les maîtrises } \\
\text { d'œuvres sociales et } \\
\text { les services techniques). } \\
\text { Assemblée générale } \\
\text { (sous l'égide de } \\
\text { l'administration): } \\
\text { validation des textes } \\
\text { constitutifs et élection } \\
\text { du bureau du comité } \\
\text { local de l'eau. }\end{array}$ & $\begin{array}{l}\text { Signature de l'arrêté } \\
\text { par l'administration } \\
\text { et session officielle } \\
\text { d'installation } \\
\text { sous son patronage. }\end{array}$ \\
\hline
\end{tabular}

Notes: le terme « administration » désigne les représentants nommés du gouvernement aux échelles départementale (préfet), provinciale (haut-commissaire) et/ou régionale (gouverneur).

*La forme de cette consultation n'est pas précisée. **'approche à suivre pour le diagnostic conjoint n'est pas précisée. ${ }^{* *}$ DGIRH (2004) n'indique pas qui rédige le projet d'arrêté. Implicitement il semblerait que cela soit une responsabilité du promoteur « obligatoirement assisté des services techniques » (DGIRH, 2004, p. 27). ${ }^{* \star \star *}$ La composition et les modalités de création de la commission ne sont pas précisées. ${ }^{* \star \star \star \star}$ Les modes de désignation ne sont pas précisés. ${ }^{\star \star \star \star \star \star A u c u n e ~ p r e ́ c i s i o n ~ n ' e s t ~ d o n n e ́ e ~ q u a n t ~ a u ~ p r o c e s s u s ~ a ̀ ~ s u i v r e ~ p o u r ~ l ' e ́ l e c t i o n ~}$ du bureau et autres structures du comité local de l'eau.

(projet d'arrêté et règlement intérieur notamment). Ces activités, cruciales dans l'optique de la mise en place d'un cadre de concertation, se sont révélées difficiles à réaliser pour l'ensemble des maîtrises d'œuvres sociales. Les acteurs présents aux réunions ont profité de l'occasion qui leur était donnée pour exprimer leurs préoccupations et, dans certains cas, leur opposition à l'inclusion d'autres usagers au sein du comité local de l'eau. Les tensions observées trouvaient leur source dans la diversité des pratiques (éleveurs-irrigants; riziculteurs-maraîchers), des localisations vis-à-vis des points d'eau (usagers amont-aval), des divisions administratives, ainsi que des appartenances politiques et sociales (autochtones-migrants; tension autour de la 
chefferie traditionnelle; membres du parti présidentiel ou opposants). Dans certains cas, la sélection de la localité où devaient se tenir les réunions a fait l'objet d'intenses tractations car l'accueil de telles réunions au caractère officiel était considéré par les acteurs comme un avantage certain pour les négociations. Pressées par le temps imparti pour mener à bien l'ensemble des activités prévues et peu préparées à faire

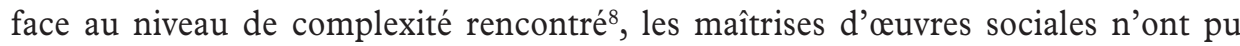
répondre à l'ensemble des attentes exprimées. Au contraire, afin de respecter leurs obligations contractuelles ${ }^{9}$, les maitrises d'œuvres sociales ont eu recours à différentes stratégies (report de réunions, recours aux forces de l'ordre, biais dans la sélection des participants pour limiter les conflits ouverts) qui ont toutes eu pour effet de mettre fin à un dialogue naissant. Elles ont élaboré les textes statutaires avec l'appui des services techniques sans réelle implication des usagers.

Le choix des participants aux différentes réunions et à l'assemblée générale constitutive au cours des étapes 3 et 4 (tabl. 1) a conduit à une dé-légitimation du processus et de la structure qui en découle (le comité local de l'eau). Aucune attention particulière n'a été portée sur ce point dans les deux guides successifs qui laissent l'initiative du choix des "représentants " aux collèges d'acteurs sans en préciser les modalités d'identification et de sélection (DGIRH, 2004, DGRE, 2010). Ces modalités ne peuvent se définir qu'en fonction du contexte local et ne sauraient donc être identifiées dans un document de cadrage à caractère national. Ce dernier pourrait cependant insister sur la nécessaire flexibilité dont il faut faire preuve et les efforts à consacrer à cette étape. Dans la pratique, et pour faire face aux contraintes financières et de temps, les maîtrises d'œuvres sociales ont presque exclusivement utilisé les canaux administratifs (i.e. les services déconcentrés du ministère de l'Agriculture, de l'Hydraulique et des Ressources halieutiques) pour identifier les participants. La sélection des représentants de l'administration, des services techniques et des collectivités territoriales pose relativement peu de problèmes car il s'agit là de groupes dont la composition est clairement établie. Pourtant, même ces choix peuvent faire l'objet de tensions, notamment entre secteurs d'activités (agriculture, élevage, environnement), entre circonscriptions (équilibre à trouver entre communes, provinces, voir entre régions), ou du fait d'appartenances politiques différentes en ce qui concerne les collectivités territoriales. Aussi est-il fréquent que les cadres de concertation multisectoriels comptent un représentant de chaque ministère pour chacune des circonscriptions administratives concernées, créant ainsi un déséquilibre et une surreprésentation de l'administration et de ses services techniques face au collège des usagers.

La situation parait encore plus complexe en ce qui concerne les usagers. Dans l'ensemble des cas documentés, aucune concertation des usagers visant à sélectionner leurs représentants n'a réellement été menée. Les maîtrises d'œuvres sociales se sont appuyées sur des relais locaux (chefs traditionnels, maires, agents de vulgarisation agricole, membres exécutifs d'organisations de producteurs) pour identifier des "usagers", en fonction de leur secteur d'activité ou de leur localisation géographique. Ceux-ci ont, par la suite, été invités par les services déconcentrés du ministère de l'Agriculture, de l'Hydraulique et des Ressources halieutiques. Cette approche a tendance à renforcer les réseaux et rapports de forces existants et à introduire un biais territorial (les participants résident souvent dans des localités à proximité des chefs-lieux où sont concentrées les administrations). Par exemple, les chefferies traditionnelles tendent à privilégier les autochtones et les propriétaires terriens par rapport aux migrants; le recours aux
8. Le choix de mettre en place des comités locaux de l'eau dans des localités où il y avait des (risques de) conflits d'usages (et donc de forts intérêts en ce qui concerne la ressource) a rendu les activités difficiles. Cependant, créer un cadre de concertation en l'absence d'enjeux clairs peut également s'avérer difficile.

9. In fine, les maîtrises $d$ 'œu res sociales étaient évaluées selon leur capacité à mener à bout le processus, c'est-à-dire à faire que des comités locaux de l'eau soient officiellement créés et reconnus par les autorités. Les termes de références s'attardaient peu sur le processus lui-même (si ce n'est l'organisation d'un certain nombre de rencontres) ou à la représentativité des acteurs qui seraient amenés à jouer un rôle au sein du place des comités locaux de l'eau. 
10. De nombreux acteurs justifient cette approche par un recours à la loi d'orientation relative à la gestion de l'eau (Gouvernement du Burkina Faso, 2001) qui identifie le ministère de I'Agriculture,

de I'Hydraulique et des Ressources halieutiques comme garant institutionnel de la gestion intégrée des ressources en eau (article 13) et

les autorités locales, dans le cadre de la collectivité ou de la circonscription administrative, comme décisionnaires (article 14). En outre, certaines maîtrises d'œuvres sociales ont indiqué qu'il s'agissait d'une consigne de la Direction générale de I'Inventaire des ressources hydrauliques, qui semble-t-il, a changé $\mathrm{d}^{\prime}$ avis depuis, soulignant le besoin et l'importance d'attribuer la présidence du comité local de l'eau à « un représentant des usagers". organisations de producteurs (qui donnent un gage d'action collective) tend à privilégier les riziculteurs des zones aménagées en aval des points d'eau plutôt que les maraîchers situés en amont qui sont plus rarement organisés en groupement.

Enfin, la désignation des membres des structures statutaires du comité local de l'eau, et notamment de son bureau exécutif, paraît cruciale (étape 4). Le bureau constitue l'organe décisionnaire du comité local de l'eau; il est responsable de l'organisation et de la tenue de réunions régulières, de la bonne conduite des activités et fait le lien entre les membres du comité local de l'eau et les autres acteurs du secteur de l'eau. L'existence, ou la nécessité, d'un bureau n'est pas explicitée dans la première version du guide (DGIRH, 2004; voir aussi tabl. 1). Dans l'esprit de l'équipe du ministère de l'Agriculture, de l'Hydraulique et des Ressources halieutiques en charge du processus au niveau national, la création d'un bureau était l'un des éléments constitutifs de l'arrêté portant " création, attributions, organisation et fonctionnement » du Comité local de l'eau devant être élaboré au cours du processus. La nouvelle version du guide (DGRE, 2010) mentionne explicitement le bureau et donne quelques éléments quant à sa composition, soulignant le besoin d'une représentation tripartite adaptée au contexte local. Le guide reste cependant très évasif quant à la procédure de mise en place de ce bureau, se limitant à l'identifier comme "élu » (DGRE, 2010, p. 44). Dans la pratique, nos enquêtes ont révélé une grande diversité de situations mais, dans tous les cas, cette étape du processus a fait l'objet d'intenses tractations sur la base des rapports de forces en présence et des appartenances sociales, politiques et spatiales des participants ainsi que de leurs activités. Les membres des bureaux ont ainsi pu être élus (généralement à main levée) à la suite d'une candidature spontanée (avant ou durant les réunions), ou sur la proposition d'un tiers ou de la présidence de l'assemblée (généralement les autorités administratives), ou simplement cooptés. Dans certains cas, les postes tels que la présidence et le secrétariat général ont été attribués d'office aux représentants de l'administration (préfet ou haut-commissaire), des services techniques (le plus souvent, le directeur provincial du ministère de l'Agriculture, de l'Hydraulique et des Ressources halieutiques) ou des collectivités territoriales (les maires) ${ }^{10}$.

La figure 2 illustre la diversité des situations quant à la représentation des différents collèges au sein des instances des comités locaux de l'eau. Aucune tendance lourde ne peut être identifiée quant à la main mise des autorités administratives sur les comités locaux de l'eau. Les usagers individuels et les organisations de producteurs sont représentés dans les bureaux des comités locaux de l'eau et certains assument des postes de responsabilité. On peut noter l'importance des services déconcentrés du ministère de l'Agriculture, de l'Hydraulique et des Ressources halieutiques au poste de secrétaire général (plus de $40 \%$ ) qui traduit leur forte implication dans le processus. Enfin, nos enquêtes font également apparaître qu'au sein d'un même comité local de l'eau les postes de responsabilité sont souvent attribués aux acteurs d'un même collège, traduisant ainsi les difficultés que les comités locaux de l'eau rencontrent dans leur rôle de cadre de concertation multipartite.

Nos enquêtes révèlent la complexité des dynamiques locales qui s'expriment dans la désignation des membres des bureaux des comités locaux de l'eau. Dans certains cas, la dévolution à l'administration et aux services techniques de la présidence et du secrétariat du comité local de l'eau perçue par les autres acteurs comme une confiscation du pouvoir par les autorités. De telles tentatives ont pu faire l'objet d'une opposition virulente aboutissant à l'exclusion de l'administration et des services techniques 
des structures décisionnaires du comité local de l'eau. Dans d'autres cas, les acteurs considèrent que ces mêmes autorités sont les mieux à même de résoudre les conflits d'usages et utiliser leur influence pour obtenir des financements. Les autorités traditionnelles semblent jouer un rôle particulier. Seuls trois des comités locaux de l'eau étudiés comptent ces leaders d'opinion dans leurs bureaux, mais ils ne sont jamais identifiés comme tels mais comme les représentants d'un secteur d'activité. Il faut également noter la faible représentation des collectivités territoriales au sein des structures décisionnaires des comités locaux de l'eau. Deux raisons à cela: la gestion des ressources en eau ne semble pas une priorité des communes (qui tendent à se focaliser sur des problématiques de développement social: éducation et santé) et les services techniques déconcentrés (au niveau de la province ou de la région) gardent le contrôle d'un processus qui reste donc très sectoriel.

En conclusion, il apparaît que la forte dynamique institutionnelle lancée au Burkina Faso n'a pas créé les conditions nécessaires à l'émergence de dynamiques endogènes d'appropriation de nouveaux espaces définis selon des limites hydrographiques, et des structures de gestion afférentes. Les usagers des ressources en eau restent en effet peu impliqués dans un processus qui reste dominé par l'administration et les services techniques du ministère de l'Agriculture, de l'Hydraulique et des Ressources halieutiques.

\section{Discussion: des institutions spatialisées mais non territorialisées}

Dans le cadre des politiques de gestion intégrée des ressources en eau, le bassin versant est vu comme un "optimum territorial» (Giraut, Antheaume, 2005). Ce positionnement nous semble cependant reposer sur un défaut de conceptualisation et de compréhension de la notion même de territoire, qui s'y trouve réduit à un concept opératoire. Invoquer le caractère «naturel» du bassin versant a en outre pour résultat (et objet selon certains auteurs) de dépolitiser la gestion des ressources en eau (pour une critique, voir Ghiotti, 2006; Warner et al., 2008).

Dans le champ de la géographie sociale, le territoire s'entend comme « appropriation à la fois économique, idéologique et politique (sociale donc) de l'espace par des groupes qui se donnent une représentation particulière d'eux-mêmes, de leur histoire, de leur singularité » (Di Méo, 1998). Cette définition permet d'apprécier la complexité de la notion et permet d'appréhender la multi-dimensionnalité du processus de territorialisation, entendu comme "l'ensemble des procédures à travers lesquelles un territoire se forme et évolue " (Turco, 1997 dans Ghiotti, 2006). Territoire et territorialisation se caractérisent notamment par une dimension descendante, politique et donc normative d'institutionnalisation de l'espace à des fins fonctionnelles, et par 
une dimension ascendante relevant du vécu, de l'idéel et des représentations (Di Méo, Buléon, 2005). Patrick D'Aquino (2002) souligne que la dimension ascendante n'est pas restreinte au domaine de l'affectif et des représentations mais doit également s'inscrire dans l'action. C'est de la fusion, difficile, de ces diverses composantes qu'émergent et se recomposent les territoires. C'est donc par l'affectif et l'action (endogène, ascendante, notamment) que le territoire diffère du concept " d'espace géographique [...] enregistrant un déficit d'appropriation et de représentations sociales, un défaut de projet " (Di Méo, Buléon, 2005), et la territorialisation de la spatialisation des politiques publiques.

Cet article analyse la mise en place de nouvelles structures de gestion de l'eau (les agences et comités locaux de l'eau) dans le cadre de la politique de gestion intégrée des ressources en eau au Burkina Faso. La démarche de création de ces structures nous apparaît être un facteur crucial de leur appropriation par les acteurs locaux, et donc de leur durabilité et de leur fonctionnement à plus long terme. Nous montrons que les dynamiques à l'œuvre au Burkina Faso relèvent plus d'une spatialisation que d'une territorialisation des politiques publiques. En effet, le changement institutionnel reste exclusivement induit par l'État (et spécifiquement par les services techniques du ministère de l'Agriculture, de l'Hydraulique et des Ressources halieutiques), centré sur des réformes publiques, et la priorité est donnée aux instruments juridiques et aux outils de gestion.

À l'échelle nationale, l'appropriation du concept de bassin versant apparaît clairement, notamment par le biais des ajustements dont il a fait l'objet, à savoir la délimitation d'" espaces de gestion " hydro-et-géo-graphiquement cohérents au sein du territoire national du Burkina Faso, et la mise en place de structures de gestion sur la base de ces limites. Dans un sens, ces espaces de gestion "font " territoire pour les décideurs politiques et leurs partenaires techniques et financiers. Cette construction territoriale n'est que peu partagée par les acteurs locaux qui n'y ont pas (ou très peu) contribué.

À l'échelle locale, la situation semble encore plus complexe. Tout d'abord, aucun consensus ne semble émerger en ce qui concerne l'échelle à considérer pour une gestion locale des ressources en eau: aménagement hydro-agricole, plan d'eau, village, commune, bassin versant sont autant de divisions de l'espace qui sont mobilisées par les acteurs en fonction de leurs objectifs et enjeux propres. Le «local» se caractérise en effet par sa multi-territorialité au sein de laquelle le bassin versant, et les institutions de gestion y afférant c'est-à-dire les comités locaux de l'eau, doivent encore trouver leur place. Même d'un point de vue institutionnel, plusieurs interprétations persistent en ce qui concerne la place et le rôle des comités locaux de l'eau et la réglementation est contradictoire. De par leurs missions et prérogatives (et notamment "l'initiation ou l'appui à toute action de développement, de promotion, de protection et de restauration du domaine public de l'eau »: DGRE, 2010) les comités locaux de l'eau apparaissent comme des instruments de territorialisation des politiques de l'eau. En outre, les "arrêtés portant création, attributions, composition, organisation et fonctionnement des CLE " les instituent en véritables associations, dotées d'une personnalité morale avec une autonomie de gestion administrative et financière (BEGE, DGRE, 2008). Le document guide de conception, création et fonctionnement des comités locaux de l'eau leur dénie cependant un tel statut en les considérant comme des " organes" des agences de l'eau auxquelles ils seraient assujettis (DGRE, 2010). En tant qu'« instances locales » des 
agences, les comités locaux de l'eau n'auraient alors que très peu de marge de manœuvre et leur rôle se limiterait à décliner localement des décisions prises par d'autres acteurs, à des échelles beaucoup plus étendues (i.e. les espaces de gestion) dans le cadre de schémas (directeurs) d'aménagement (SAGE et SDAGE) qui restent à définir.

Cette situation institutionnelle hybride a des conséquences en termes d'action. Certains acteurs restreignent les comités locaux de l'eau à un rôle de sensibilisation, d'autres envisagent qu'ils puissent décider et piloter de véritables projets d'aménagement du territoire (la protection des berges étant l'exemple le plus courant). Dans ces conditions, il paraît donc normal que l'élaboration d'outils de gestion (comme la réglementation l'envisage: DGRE, 2010), relève de la gageure (sur les treize comités locaux de l'eau documentés, seuls trois ont été accompagnés dans la création d'un plan de gestion $)^{11}$. Mais cela n'est pas forcément négatif: en l'absence de vision partagée, de tels outils de gestion ne pourraient être développés que de façon descendante, sans créer les conditions d'une action endogène. À l'inverse de l'approche actuelle (et justifiée par le besoin de visibilité vis-à-vis des partenaires techniques et financiers), il nous semble que de tels outils de gestion se doivent d'être conçus comme l'aboutissement d'un processus de territorialisation (plutôt que son préalable), et ce, sur des espaces socialement construits à l'inverse de zones de compétence délimitées de façon technocratique.

L'incertitude en ce qui concerne les attributions des comités locaux de l'eau constitue également un frein à leur légitimation notamment vis-à-vis des usagers, des représentants des collectivités territoriales et autres organisations sectorielles et spatiales. La question de la légitimité, centrale à tout processus de territorialisation, est l'un des défauts majeurs de l'approche suivie pour la mise en place des comités locaux de l'eau. Cette dernière, s'inscrivant dans un temps court (trois mois), n'a pu créer les conditions d'une représentation et d'une représentativité partagées. Certes les comités locaux de l'eau sont pourvus de bureaux exécutifs généralement multipartites et se réunissant plus ou moins régulièrement (et dans certains cas, d'autres structures d'appui: conseil technique, conseil des sages) mais ceux-ci, quand ils fonctionnent, semblent le faire en vase clos, sans lien avec les acteurs qu'ils sont censés représenter. En témoignent l'absence d'assemblées générales convoquées sur initiative locale et l'aveu, général de la part des membres des bureaux des comités locaux de l'eau, d'une absence de mobilisation des acteurs. Ces derniers se retrouvent dans d'autres forums (organisations paysannes, associations, comités villageois) dont l'articulation avec le comité local de l'eau n'a pas été suffisamment pensée. Le bassin versant n'est donc pas doté d'une organisation pouvant agir en son nom; il n'émerge pas en tant que nouveau territoire de l'eau.

\section{Conclusion}

Dès le milieu des années 1990, le Burkina Faso a lancé une dynamique institutionnelle et politique visant à promouvoir une gestion intégrée des ressources en eau. Le manque d'implication et d'adhésion des acteurs, et notamment des utilisateurs des ressources en eau, est aujourd'hui identifié comme l'une des raisons principales des retards que les réformes enregistrent. La mise en place de nouvelles structures de gestion épousant les limites hydrographiques des bassins versants fait particulièrement débat, mais sans que l'approche qui préside à leur mise en place soit questionnée.
11. Deux de ces trois comités locaux de l'eau ont par la suite bénéficié de l'appui de partenaires techniques et financiers de ces plans de gestion. Les autres comités locaux de l'eau ont, à partir de 2008, obtenu des subventions de l'État pour des actions ponctuelles de protection des berges. Ces subventions se sont rapidement épuisées; la gestion de fonds publics étant en ellemême au-delà des compétences des membres des comités locaux de l'eau (entretien avec un membre du SP/PAGIRE basé à Ouagadougou). À I'heure actuelle, I'agence de l'eau du Nakambé appuie les comités locaux de l'eau existants dans son espace de gestion par le biais des services techniques du ministère de l'Agriculture, de l'Hydraulique et des Ressources halieutiques pour l'élaboration de plans d'action annuels, sur la base desquels des financements pourront être alloués. Le niveau réel d'implication des membres des comités locaux de l'eau (et notamment des représentants des usagers) vis-à-vis des services techniques déconcentrés reste cependant à déterminer. 
Remerciements.

Les auteurs remercient Pierre Zoungrana et Dr Fulgence Ki du SP/PAGIRE pour leur précieuse collaboration sans laquelle il ne nous aurait pas été possible d'apprécier les dynamiques de la gestion intégrée des ressources en eau au Burkina Faso.

Nous remercions également Francine $\mathrm{Ki}$, Kassoum Ouedraogo, Korotimi Sanou, et Gael Ndanga Kouali pour leur assistance.

Cette recherche a été menée grâce au soutien du Challenge Programme for Water and Food (CPWF) dans le bassin de la Volta, de I'International Water Management Institute (IWMI), du CIRAD et de l'université de Wageningen. Nous remercions enfin les trois relecteurs anonymes et le comité éditorial de la revue pour leurs conseils avisés ayant permis d'améliorer nos arguments.
Les réformes et politiques de gestion intégrée des ressources en eau mises en place au Burkina Faso mettent les structures étatiques au centre du changement; elles ne permettent pas l'expression des priorités des usagers et l'émergence d'actions locales ascendantes. D'un point de vue géographique, cette approche institutionnelle et fonctionnaliste relève d'une spatialisation de la politique et de la gestion de l'eau, et non de leur territorialisation dans la mesure où les acteurs locaux (les usagers notamment) n'attribuent aucune identité à l'espace du bassin versant. L'approche actuelle met l'accent sur l'identification et le tracé de limites (espaces de gestion, zones de compétence), au sein desquelles action et mobilisation acquerraient une nouvelle légitimité dans le cadre d'organes de gestion créés pour l'occasion (agence de l'eau et comités locaux de l'eau). Cette insistance à délimiter de nouveaux espaces est un obstacle à l'émergence et l'expression de dynamiques endogènes, notamment dans un contexte africain où "les territoires sont assez flous et ne semblent pas s'arrêter à une figure aisément cartographiable selon les principes de l'exclusivité et de l'exhaustivité des limites " (Retaillé, 2005, p. 179). En «naturalisant " le bassin versant et, ce faisant, dépolitisant la gestion des ressources en eau, les politiques de gestion intégrée des ressources en eau vont à l'encontre d'une réelle construction territoriale pourtant nécessaire dans une perspective de durabilité et d'équité.

\section{Références}

D’Aquino P. (2002). « Le territoire entre espace et pouvoir: pour une planification territoriale ascendante ». L'Espace géographique, t. 31, nº 1, p. 3-22.

Blomquist W., Schlager E. (2005). "Political pitfalls of integrated watershed management ». Society and Natural Resources, vol. 18, nº 2, p. 101-117.

Bouquet C. (2012). «La GIRE: Un modèle difficile à transférer en Afrique sub-saharienne ». In JuLIEN F. (dir.), La Gestion intégrée des ressources en eau en Afrique subsaharienne. Paradigme occidental, pratiques africaines. Québec: Presses de l'Université du Québec, 306 p.

Bureau d'étude des géosciences et de l'environnement (Bege), Direction générale des Ressources en eau (DGRE)(2008). Mission d'évaluation des processus de mise en place des comités locaux de l'eau et de leur opérationnalité. Ouagadougou: Direction générale des Ressources en eau, $106 \mathrm{p}$.

CHÉNÉ J.-M. (2009). «Introduction - Integrated water resources management: Theory versus practice ». Natural Resources Forum, vol. 33, n 1, p. 2-5.

Cherlet J., VenOt J.-P. (2013). «Structure and agency: Understanding water policy changes in West Africa ». Water Policy, vol. 15, nº 3, p. 479-495.

Dı MÉo G. (1998). Géographie sociale et territoires. Paris: Nathan Université, 320 p.

Dı MÉo G., BulÉon P. (dir.)(2005). L'Espace social. Une lecture géographique des sociétés. Paris: Armand Colin, $303 \mathrm{p}$.

Direction générale de l'Inventaire des Ressources hydrauliques (Dgirh)(2004). Les Comités locaux de l'Eau (CLE) maillons de base du cadre institutionnel de gestion intégrée des ressources en eau du Burkina Faso. Ouagadougou: Gouvernement du Burkina Faso, 36 p.

Direction générale des Ressources en eau (Dgre)(2006). État de la mise en œuvre du plan d'action pour la gestion intégrée des ressources en eau (PAGIRE) du Burkina Faso, mars 2003-juin 2006. Ouagadougou: Gouvernement du Burkina Faso, 25 p. 
Direction générale des Ressources en eau (Dgre)(2010). Les Comités locaux de l'eau (Cle) maillons de base du cadre institutionnel de Gestion Intégrée des Ressources en Eau du Burkina Faso. Ouagadougou: Gouvernement du Burkina Faso, 57 p.

Direction GénÉrale des Ressources en EAU (DGRe), Danida, Asdi (2008). Revue tripartite à mi-parcours de la composante "Appui au PAGIRE » Programme d'appui au développement du secteur Eau et Assainissement (PADSEA II) 2005-2009. Ouagadougou: Gouvernement du Burkina Faso, 103 p.

Gouvernement du BuRkina FASO (GdBF)(2001). Loi no 002-2001/AN portant loi d'orientation relative à la gestion de l'eau. Ouagadougou: Gouvernement du Burkina Faso, $18 \mathrm{p}$.

Gouvernement du BuRkina FASO (GdBF)(2003). Plan d'action pour la gestion intégrée des ressources en eau (PAGIRE). Ouagadougou: Gouvernement du Burkina Faso, 74 p.

Giraut F., Antheaume B. (2005). «Introduction. Au nom du développement, une (re)fabrication des territoires ». In Antheaume F., Giraut B. (dir.), Le Territoire est mort Vive les territoires! Une (re)fabrication au nom du développement. Paris : IRD Éditions, p. 9-36.

GніотІ S. (2006). «Les territoires de l'eau et la décentralisation. La gouvernance de bassin versant ou les limites d'une évidence ». Développement durable et territoires, dossier 6 : Les territoires de l'eau. http://developpementdurable.revues.org/1742

GRAefe 0. (2011). « River Basins as new environmental regions? The depolitization of water management ». Procedia - Social and Behavioral Sciences, vol. 14, p. 24-27.

Global Water Partnership (GWP). (2000). La Gestion intégrée des ressources en eau. Stockholm: Global Water Partnership, TAC Background paper no. 4, $80 \mathrm{p}$.

Global Water Partnership/Afrique de l'ouest (GWP/AO)(2009). Capitalisation du processus d'élaboration du Plan d'action de gestion intégrée des ressources en eau (PAGIRE) et de sa mise en œuvre au Burkina Faso. Ouagadougou: Global Water Partnership/Afrique de l'ouest, 52 p.

LANKFORD B., HEPWORTH N. (2010). «The cathedral and the bazaar: Monocentric and polycentric river basin management ». Water Alternatives, vol. 3, n 1 , p. 82-101.

Ministère de l'Agriculture, de l'Hydraulique et des Ressources Halieutiques (mahrh). (2010). Revue conjointe 2010 de la mise en cuvre du programme national d'approvisionnement en eau potable et d'assainissement (PN-AEPEA) et du plan d'action pour la gestion intégrée des ressources en eau (PAGIRE). Ouagadougou: Ministère de l'Agriculture, de l'Hydraulique et des Ressources halieutiques, rapport du groupe thématique 4, $13 \mathrm{p}$.

Molle F. (2012). «La GIRE: anatomie d'un concept ». In JuLIEN F. (dir.), La Gestion intégrée des ressources en eau en Afrique subsaharienne-Paradigme occidental, pratiques africaines. Québec: Presses de l'Université du Québec, p. 23-53.

PetT O., BARon C. (2009). «Integrated water resources management: From general principles to its implementation by the state. The case of Burkina Faso ». Natural Resources Forum, vol. 33, $n^{0} 1$, p. 49-59.

Rétalllé D. (2005). «L'espace mobile ». In Antheaume F., Giraut B. (dir.), Le Territoire est mort Vive les territoires! Une (re) fabrication au nom du développement. Paris: IRD Éditions, p. 175-201.

SHAH T., van Koppen B. (2006). "Is India ripe for integrated water resources management? Fitting water policy to national development context ». Economic and Political Weekly, 5 août, p. 34133421.

TuRCo A. (1997). «Aménagement et processus territoriaux: l'enjeu sémiologique». Espaces et sociétés, nº 90-91, p. 231-249.

VENOT J.-P., HiRVONEn M. (2013). «Enduring controversy: Small reservoirs in Sub-Saharan Africa ». Society and Natural Resources, vol. 26, nº, p. 883-897.

Warner J., Wester P., Bolding A. (2008). « Going with the flow: River basins as the natural units for water management? ». Water Policy, vol. 10, supplément 2, p. 121-138. 